\title{
Preferencias en Modalidades de Clases y sus Herramientas
}

\section{Preferences in Class Modalities and their Tools}

\author{
Karla Aguilera Moreno \\ Escuela Superior Politécnica del Litoral \\ Guayaquil, Ecuador \\ karamore@espol.edu.ec \\ Orcid: 0000-0003-0811-6887
}

\author{
Génesis Quiroz Calderón \\ Escuela Superior Politécnica del Litoral \\ Guayaquil, Ecuador \\ gquiroz@espol.edu.ec \\ Orcid: 0000-0002-9153-8222
}

\author{
Elizabeth Elizalde Ríos \\ Escuela Superior Politécnica del Litoral \\ Guayaquil, Ecuador \\ eselizal@espol.edu.ec \\ Orcid: 0000-0001-9763-4306
}

\begin{abstract}
Resumen - El uso de las pizarras virtuales, hoy en día, es una forma de tecnología de la información y comunicación muy utilizada para impartir conocimiento; de igual manera, existen diferentes plataformas que combinan la implementación de la pizarra virtual con juegos educativos. Esto es muy útil sobre todo en la educación en línea en donde ha tenido un incremento en los últimos años. El objetivo de este estudio es comparar el método de educación tradicional y la digital causado por la emergencia del Covid19, con el fin de conocer las ventajas de las herramientas digitales en el proceso de aprendizaje de niños del nivel básico inicial de la provincia del Guayas. Además, identificar la efectividad de estas herramientas y establecer su importancia. Se utilizó un enfoque metodológico filosófico pragmático y el método mixto que combina los métodos cualitativos y cuantitativos. Los resultados de esta investigación señalan que la mayoría de los sujetos tienen preferencia por la modalidad digital, sin embargo, existen varias dificultades que provocan cierto rechazo a utilizar este método.
\end{abstract}

Palabras Clave: Pizarras virtuales, Tecnología, Educación, Juegos.

\begin{abstract}
Nowadays, the employment of virtual whiteboards is a widely used information and communication technology to impart knowledge; similarly, there are various platforms that combine the implementation of the virtual whiteboard with educational games. This is very useful especially in online education which has had an increase in recent years. The objective of this study is to compare traditional and digital education methods caused by the Covid19 emergency, to know the advantages of digital tools in the learning process of children in Initial Basic Level in the Guayas province. Also, to identify the effectiveness of those tools and establish their importance. A pragmatic philosophical methodological approach and the mixed method were used, combining qualitative and quantitative methods. The results of this research indicate that most of the subjects prefer the digital modality, however, there are several difficulties that cause certain rejection to use this method.
\end{abstract}

Keywords: Virtual whiteboards, Technology, Education, Games.

Sumario: I Introducción, II Metodología, III Resultados y Discusión, IV Conclusiones.

Como citar: Aguilera, Karla., Quiroz, Génesis., Elizalde, Elizabeth. (2020). Preferencias en Modalidades de Clases y sus Herramientas. Revista Tecnológica - Espol, 32(2). Recuperado a partir de http://www.rte.espol.edu.ec/index.php/tecnologica/article/view/771 


\section{INTRODUCCIÓN}

\section{A. Antecedentes}

En el más reciente boletín epidemiológico que ofrece el Ministerio de Salud [1], se establece que Ecuador tiene 135.749 pacientes con coronavirus comprobados por medio de la prueba del PCR. En Guayas, se encuentran 20.365 de los casos encontrados en Ecuador. Debido a la pandemia mundial del virus COVID19, los planteles estudiantiles han cerrado sus puertas y han empezado una etapa de educación a través del Internet. Para el caso del Ministerio de Educación, el programa Aprendamos juntos en casa delineó las guías para el trabajo de los profesores para el año lectivo 2020 [2]. En Ecuador, las instituciones educativas se encuentran divididas según su sostenimiento, en $12.409(76,06 \%)$ instituciones fiscales, $552(3,41 \%)$ instituciones fiscomisionales, $115(0,71 \%)$ municipales y $3.124(19,28 \%)$ particulares [3].

El objeto de estudio se centra en la región Costa, provincia del Guayas, donde 1.567 instituciones son fiscales, 34 fiscomisionales, 13 municipales y 1.043 particulares, con 1'076.446 estudiantes y 46.948 docentes para toda la provincia [3].

En el contexto de pandemia, la modalidad de clases en línea no es una opción, sino la regla. Enrique Dans [4] habla de que la educación, a través del internet ya sea de modo parcial o completa, ha experimentado un gran crecimiento en los últimos años. Esta situación ocurre porque es más fácil y efectivo transmitir determinados tipos de conocimientos en la modalidad en línea, específicamente aquellos que necesitan una interacción entre profesor-alumno o entre alumnos [5]. Si bien esto aplica para estudiantes autónomos, el panorama varía para los niños más pequeños que requieren control parental, pues se hace más evidente la limitación de infraestructura $\mathrm{y}$ recursos en los procesos de enseñanza-aprendizaje, como la habilidad de escritura, al restar afectividad, tiempo y práctica a la incorporación de estas herramientas [6].

Tanto en los ámbitos teórico y práctico, han surgido muchas dudas respecto al uso de estas herramientas en la educación, debido a que, para muchas instituciones y docentes representan un reto. Sin embargo, es labor de las instituciones y los docentes estar a la vanguardia de los cambios y nuevas tecnologías disponibles para poder aprovecharlas y usarlas a favor de los alumnos y de ellos.

A medida que la red se actualiza con tendencias y los alumnos cambian su manera de relacionarse con su ambiente, los esquemas y plataformas educativas también deberían hacerlo [5].

Ahora que las instituciones académicas han cerrado su infraestructura física, los profesores deben buscar una manera de atraer la atención de sus alumnos, día a día en sus clases virtuales. En esta nueva modalidad se resalta la creatividad y mayor preparación de las partes involucradas: maestros y estudiantes. La directora de la International School, Mercedes Sánchez afirma que:

"La educación virtual nunca igualará a la presencial, sin embargo, el compromiso adquirido puede lograr grandes cambios y el que quiere, puede. (...) Sea la educación virtual o presencial, lo importante es atravesar esta adversidad superando los obstáculos y nunca descuidando la calidad." [7]

Unos de estos ápices para desarrollar la creatividad son las pizarras digitales en línea. Desde el 2016 han existido varios artículos [8] recomendando esta herramienta a estudiantes. $\mathrm{Su}$ objetivo inicial fue realizar trabajos en grupo. Estas son descritas como necesarias para compartir ideas libremente, ya que, cuando se realiza trabajos en equipo en la modalidad presencial, muchas veces falta el sentido del orden. A su vez, sirven para dibujar con pinceles y lápices digitales y se asemeja a una pizarra normal. También las utilizan para ubicar los puntos principales de una sesión de clases, o ideas emergentes. No es utilizada como una única forma de dar clases, sino como un recurso del e-learning.

También, un recurso novedoso que se está utilizando para la educación en línea es la inserción de los juegos educativos [9]. Tienen en común la pizarra virtual, el uso de una pantalla digital, en donde actúan como medio de aprendizaje ya que los usuarios pueden adquirir conocimiento al interactuar con estos [10]. Los juegos educativos contienen elementos de motivación necesarios para captar la atención de los usuarios, que, junto a los objetivos de aprendizaje, son un resultado de las actividades que se realizan en la dinámica del juego. En este tipo de juegos existen características y contextos de uso, que se pueden modificar para que se vuelvan más efectivos a la hora de cumplir el objetivo de enseñanza, debido a que cada usuario tiene diferentes preferencias en cuanto a lo visual y a lo didáctico [11].

\section{B. Justificación}

En el contexto académico y tecnológico actual, los profesores se encuentran en un nuevo medio, en un nuevo ciclo del aprendizaje, en donde las nuevas tecnologías están en auge y deben motivarse a ser manejadas. La creatividad e independencia es un eje fundamental que se maneja en esta área [12]. Harold Villalva Melgar, investigador tecnólogo, explica sobre la situación actual, no solo en general, sino también en el sector educativo:

"Durante este confinamiento mundial las tecnologías y el Internet han sido herramientas muy útiles a la hora de hacer teletrabajo, capacitaciones, transacciones, pedidos de alimentos e insumos, reuniones de familiares y amigos, entre otras actividades. En el área escolar se ha masificado el uso de las aplicaciones de videoconferencia ante la necesidad y adaptación para este nuevo cambio y aún más en nuestro medio que era totalmente desconocido o poco usada." [12]

Por ende, las modalidades de enseñanza han cambiado, como el paso a la modalidad en línea, que utiliza dispositivos como medio de comunicación, para la cual los maestros deben prepararse y no perder la atención de los estudiantes.

Una de las herramientas utilizadas en esta modalidad son las pizarras digitales, que provocan interacción y un cambio total en el dinamismo de la clase. Así señala un 
ingeniero en sistemas y capacitador a profesores, Stalin Ludeña:

“También hay otras como las pizarras digitales. Por ejemplo, un profesor de Ciencias Naturales quiere explicar algo sobre el esqueleto humano, tú pones la imagen y la compartes con todos los alumnos, y tú como maestro vas a decir: 'Quién me puede explicar en qué parte del esqueleto humano está el fémur'; entonces, en línea, automáticamente el alumno lo va a hacer y a compartirlo con todos, va a indicar dónde está ubicado." [13]

Las herramientas digitales, propias de las Tecnologías de la Información y la Comunicación (TICs), se ejecutan con ayuda del profesor en el aula. En estudios realizados sobre el uso de estas herramientas en la educación para niños, la recepción del nivel de conocimiento ha sido considerado como bueno, pudiendo mejorar la curva de aprendizaje de la herramienta [14].

Por lo que es primordial la investigación sobre estas herramientas innovadoras para poder conocer las diferencias que causan en el aprendizaje de niños, en comparación con los métodos tradicionales de elaborar una clase.

\section{Problema}

Los niños que están asistiendo a la educación formal, en un entorno de aislamiento, son parte de una generación tecnológica, por lo que los métodos tradicionales podrían no ser totalmente efectivos a la hora de impartir conocimientos por parte de los docentes. Los juegos educativos online en conjunto a las pizarras digitales se presentan como posibles herramientas de aprendizaje que romperían el molde tradicional y mejoraría la eficacia del aprendizaje en los niños.

En el caso de la problemática, esta investigación se enfoca en las ventajas y desventajas de este tipo de herramienta tecnológica y digital, su aplicación en juegos interactivos frente a otros métodos tradicionales, y cómo se podrían utilizar para la ejecución de una clase más interactiva y efectiva [15].

\section{Objetivos}

\section{1) Objetivo General}

- Determinar las ventajas de las herramientas digitales en el proceso de aprendizaje de niños del nivel básico inicial de la provincia del Guayas, en comparación a los métodos tradicionales, para la comprensión de resultados.

2) Objetivos Especificos

- Identificar la efectividad de las herramientas digitales para el análisis de casos en niños que cursan el nivel básico inicial.

- Establecer la importancia de las herramientas digitales con carácter lúdico para su implementación en la educación de niños del nivel básico inicial.

\section{E. Pregunta de Investigación}

¿Cuáles son las ventajas que tienen las pizarras virtuales que se utilizan en juegos interactivos en el proceso de aprendizaje de niños de nivel básico inicial de la provincia del Guayas, frente a los métodos tradicionales?

\section{METODOLOGÍA}

\section{F. Estado del Arte: Juegos Educativos}

Según los autores Carro, Breda y Castillo [11], la unidad básica de un juego educativo es la actividad, que se puede desarrollar exitosamente mediante la identificación de objetivos (conocimiento final que adquiere el usuario), tipo (educativa / no educativa) y dificultad (fácil/difícil).

Por ejemplo, ÁrbolABC.com, una página web que contiene gran diversidad de juegos educativos divididos en categorías de edades, con un diseño visual adecuado para llamar la atención de niños que cursan la educación primaria, también existe material extra para que los niños se diviertan desarrollando distintas destrezas. Los juegos educativos se vuelven didácticos y funcionales si el estudiante o usuario tiene participación directa con el material de estudio, es por esto por lo que las pizarras virtuales juegan un papel importante a la hora de obtener un modelo de juego educativo ideal, y ese es el tema en el que se enfoca esta investigación [16].

Las áreas involucradas en su desarrollo y utilización son: la educativa, tecnológica y de diseño. Estas justifican la aplicación de las pizarras, y su impacto es una de las aristas de este proyecto, ya que están directamente involucrados los estudiantes, profesores y centros educativos. Los niños están expuestos a la tecnología y se empiezan a relacionar con esta desde temprana edad. En el área de diseño, se involucra a los diseñadores gráficos en la creación y análisis de composiciones visuales efectivas en las pizarras digitales y juegos para propiciar mejores resultados en la interacción del estudiante con los temas educativos [17].

Por medio de la investigación en el diseño, Findeli [18] indica que esta aporta a desarrollar los conocimientos en áreas como la informática, diseño de aplicaciones digitales, y principalmente ciencias naturales y sociales, estas últimas son materias impartidas a los estudiantes de educación primaria.

Las Tecnologías de la Información y la Comunicación (TICs) han sido utilizadas con tres diferentes objetivos en los ambientes de aprendizaje:

- Apoyar la transmisión de mensajes a los estudiantes por medio de tutoriales, ejercitadores y sitios web informativos

- Apoyar el aprendizaje activo mediante la experimentación con los objetos de estudio a través de simuladores de procesos.

- Facilitar la interacción para aprender mediante juegos en red colaborativos, mensajería electrónica, correo electrónico, foros, video o audio conferencia [19].

Por otra parte, Fouts [20] clasificó a las TICs de acuerdo con sus propósitos educativos. Los cuales con: Enseñar, practicar y ejercitar, simular, resolver problemas y elaborar 
productos, proveer acceso a la información, y servir como medio de comunicación con otras personas.

Las pizarras virtuales se han convertido en una forma de TIC [21] para impartir conocimientos. Su desarrollo y aplicación en los juegos educativos infantiles ha permitido que el desenvolvimiento y comprensión estudiantil aumente, así también el interés de los escolares en otras áreas del conocimiento. Así mismo, el uso y manejo de estas ha provocado que el profesorado adquiera nuevas habilidades. Para esto es necesario un correcto diseño de los juegos educativos y que el GUI (Graphical User Interface, por sus siglas en inglés) cumpla con los principios del diseño que son: la familiaridad del usuario, interfaz uniforme, mínima sorpresa, recuperación de estados, guía de usuarios, diversidad de usuarios, adoptar el punto de vista del usuario, realimentación, potenciar la sensación de control del usuario sobre el sistema, minimizar la necesidad de memorización, anticipación, percepción de color y tamaño, legibilidad, valores por defecto y eficiencia.

\section{G. Educación Tradicional y Nuevas Tecnologías}

El mundo se está transformando, y el sistema educativo debe adaptarse al avance que ha tenido la sociedad, los conocimientos y las nuevas tecnologías. La Organización Mundial de la Salud (OMS) diferencia entre el tiempo que el niño pasa solo frente a la pantalla y el tiempo que pasa frente a una pantalla realizando alguna actividad tranquila que ayude en su desarrollo intelectual, a este tiempo lo llaman "tiempo de calidad". Sin embargo, según la OMS se recomienda que los niños entre dos y cinco años no pasen más de una hora frente a una pantalla y que este tiempo, frente a la pantalla, sea de calidad [22].

En una entrevista realizada por Myriam López para la revista Kindsein en 2007, Schank dice que: "El sistema educativo que tenemos hoy y que ha seguido invariable desde hace años se puede resumir de la siguiente manera: un profesor entra en clase y habla. Los alumnos, como mucho toman apuntes y como no pueden recordar lo que se les dijo, les hacen exámenes. Pero poco después de hacer los exámenes lo olvidan todo" [23]. Si ahora mismo se les tomara el examen de historia que rindieron en años anteriores no podrán aprobarlo. Una razón de esto es que la educación tradicional tiene como objetivo que los estudiantes aprueben sus exámenes y tengan buenas notas. Hoy en día los estudiantes tienen intereses diferentes y aprenden de una forma más interactiva, por lo que es preciso modificar estos métodos tradicionales [23].

Es importante recordar que todos tenemos estilos diferentes de aprendizaje, por lo que estas nuevas tecnologías ayudan a crear micro mundos, en donde el alumno tiene estas herramientas que puede utilizar independientemente, permitiendo así que se involucre en su propio proceso de enseñanza. Según Robinson [24], como desventaja hay que recalcar, que esto supone, cambios en las funciones cognitivas, en el objetivo del aprendizaje y además de la constante capacitación de los profesores en el área tecnológica, así como tener la disponibilidad de insumos tecnológicos. El componente más favorecedor pero desventajoso de esta modalidad, es que su éxito recae en la integración adecuada de la tecnología en el plan de estudio. Ecuador, siendo un país en vías de desarrollo, aún no está preparado para esto debido a la carencia que existe tanto en equipos tecnológicos, como en el conocimiento para el uso de estas herramientas.

\section{H. Estado del Arte: Casos de estudio}

Siguiendo el lineamiento de entender más sobre el tema, se presenta como un ejemplo, el caso de estudio de la Universidad de Otavalo. En el cual, se realizó encuestas a 25 docentes y a una muestra estratificada de los estudiantes sobre el uso de pizarras digitales. En esta, se obtuvieron los siguientes resultados: las pizarras digitales sí optimizan el tiempo de enseñanza según el profesor, pero se muestra una sobreestimación leve de parte de los profesores en el beneficio del entendimiento del tema y el aumento de la interacción en clase, en comparación a la obtenida por los estudiantes. Además, según profesores, estas herramientas sí promueven la innovación didáctica [25].

Otro caso de estudio que trata de demostrar la autoeficacia y motivación de estas herramientas se dirigió a estudiantes con discapacidad en un rango de edad de 6 a 21 años. Este caso constituyó un estudio fenomenológico y de observación, en donde, al usar pizarras digitales, en conjunto a juegos didácticos, se mostró un mayor grado de atención, mayor motivación y disfrute de actividades. Esto se corrobora porque se grabaron los dos tipos de clases con los dos métodos: tradicional y digital, y se observó estas diferencias primordiales. También, se realizaron entrevistas, y se obtuvo resultados favorables en áreas de motivación, interacción y autoeficacia. En este caso, se remarcó la infinidad de recursos en línea, pero así su contraparte de la necesidad de tener la disponibilidad de herramientas tecnológicas y profesores capacitados [26].

Se puede comprender más sobre el tema, gracias a estos casos de estudio de cómo profesores de educación básica empezaron a insertar el uso de pizarras digitales en sus clases. Estos estudios resaltan la importancia del constante aprendizaje del profesor sobre estas herramientas, en donde, ellos son los agentes del cambio en la clase y en los estudiantes. En el estudio del 2010, participaron tres profesores por medio de grabaciones de sus clases por dos a tres horas, así como en observaciones no participativas, entrevistas y apuntes. En conclusión, se elaboró un estudio exhaustivo sobre las herramientas utilizadas por los profesores y se encontró varias lagunas en el manejo de estas herramientas y la forma cómo las controlaban en su respectiva clase. Por medio de falla y error, preguntar a colegas, preguntar a los estudiantes para así desarrollar su perspectiva crítica de las situaciones, se llegó a la conclusión de que se necesita un reforzamiento constante a los profesores para que las pizarras digitales tengan el efecto positivo deseado [27].

\section{Enfoque Metodológico}

Para el desarrollo de esta investigación se utilizó un enfoque metodológico filosófico pragmático y el método mixto, el cual tiene como principal característica la combinación de los métodos cualitativos y cuantitativos. La unión de estos métodos permite obtener conocimiento que no se podría obtener en forma separada, pues relaciona 
criterios y descriptores obtenidos por las herramientas aplicadas.

El método mixto permite que el investigador tenga mayor confianza en los resultados obtenidos [28], ya que, al combinar ambos métodos, los resultados de uno se pueden complementar con los resultados del otro método. Obteniendo respuestas más significativas y que aportan más en la comprensión del fenómeno estudiado.

Se escogió este método por ser el que mejor se adapta a las necesidades de la investigación. Los datos cuantitativos obtenidos permiten identificar la relación que existe entre las variables cuantificadas, Por otro lado, los datos cualitativos recolectados facilitan el proceso de entender e identificar las realidades que son datos más profundos.

\section{J. Herramientas, Recolección y Análisis de Datos}

Debido al enfoque de esta investigación y con el fin de obtener los mejores resultados posibles, se planificó utilizar dos estrategias de recolección de datos, uno basado en el método empírico [29] y otro en el estadístico [30]. Estas estrategias fueron aplicadas a la muestra de 55 estudiantes que cursan la educación primaria y a 24 profesores, que se seleccionaron de acuerdo al criterio que asistan o enseñen en los niveles básicos iniciales de instituciones educativas. Los sujetos fueron ubicados por teléfono y referencias personales. Se aplicaron estas estrategias, debido a que ellos son los que proporcionarían información relevante para la investigación.

Siguiendo el método empírico se hizo uso de entrevistas como herramienta para recolectar los datos. Se realizó una entrevista semiestructurada ya que se les dio la posibilidad a los entrevistados para que pudieran expresar su punto de vista libremente. Para las entrevistas, los sujetos seleccionados fueron veinticuatro profesores de diferentes instituciones educativas. Estas entrevistas por medios y plataformas virtuales demostraron la verdadera postura de los profesores sobre la implementación de esta herramienta digital en las aulas de clases, además permitieron entender cuál es el impacto que tiene esta herramienta en el aprendizaje de los niños.

Por otro lado, siguiendo el método estadístico se diseñaron encuestas. Con el fin de obtener resultados adecuados se realizaron dos modelos de encuestas. En la encuesta dirigida a los profesores se les preguntó sobre las herramientas digitales que utilizan para sus clases y cuáles son sus ventajas. Se encuestó a veinticuatro profesores en total. Esta encuesta estaba compuesta de preguntas cerradas y siguiendo las siguientes escalas: dicotómicas, ordinal politómica, continua y nominal politómica. Realizar las preguntas siguiendo estas escalas permitió realizar la cuantificación de los datos de una manera más fácil ya que los datos son exactos.

En cuanto a la encuesta dirigida a un total de cincuenta y cinco niños, se les preguntó sobre el uso de las herramientas digitales y el impacto que tiene sobre su aprendizaje. La encuesta estaba compuesta de preguntas cerradas y con las siguientes escalas: dicotómicas, ordinal politómica y nominal politómica. Estas preguntas estaban redactadas de forma que los niños pudieran entenderlas con facilidad. Sin embargo, la participación de los padres de familia fue vital para la aplicación de la herramienta.
Al finalizar con el proceso de recolección de datos se procedió a analizar la información obtenida. Las entrevistas proporcionaron términos claves que permitieron realizar un análisis profundo sobre el tema y conocer las opiniones de los sujetos. Las encuestas por otra parte brindaron datos estadísticos que permitieron categorizar las variables y cuantificar los resultados. Al finalizar, se comparó las respuestas obtenidas entre ambos métodos y se determinó criterios en la conclusión.

\section{RESULTADOS Y DISCUSIÓN}

Al utilizarse el método mixto, se obtuvo algunos resultados de dos áreas. Por una parte, las entrevistas a docentes $\mathrm{y}$, por otra parte, las encuestas a estudiantes $\mathrm{y}$ docentes.

\section{K. Entrevistas}

Las entrevistas fueron realizadas a docentes por medio de las plataformas de Zoom y WhatsApp. En esta sección, se detalla acotaciones realizadas por los diferentes profesores, asociadas a términos generales para su mayor entendimiento. Ver TABLA I

TABLA I

VENTAJAS SEGÚN PROFESORES

\begin{tabular}{|c|c|}
\hline Términos & Citas \\
\hline Facilidad & $\begin{array}{l}\text { "Nos facilita que ellos entiendan } \\
\text { muchas cosas que tal vez pueden ser } \\
\text { difíciles de entender". } \\
\text { "Característica como la accesibilidad, } \\
\text { bajo costo y fácil manejo son las que harán } \\
\text { de los recursos digitales una herramienta } \\
\text { dinámica". }\end{array}$ \\
\hline Interacción & $\begin{array}{l}\text { "Son herramientas que me han } \\
\text { facilitado bastante las sesiones de clases, } \\
\text { me han permitido tener una buena } \\
\text { interacción con los estudiantes". } \\
\text { "Entonces para ellos es ameno contar } \\
\text { con los recursos digitales porque es una } \\
\text { forma interactiva de implementar en los } \\
\text { estudiantes el aprendizaje perdurable". } \\
\text { "Los estudiantes han mostrado mayor } \\
\text { nivel de interacción entre ellos". }\end{array}$ \\
\hline Dinamismo & $\begin{array}{l}\text { "La clase sea divertida y más dinámica } \\
\text { para ellos". }\end{array}$ \\
\hline Entendimiento & $\begin{array}{l}\text { "Los niños se adaptan muy bien al tema } \\
\text { virtual". } \\
\text { "Los niños aprenden por lo visual". }\end{array}$ \\
\hline Aprendizaje & "Los niños aprenden por lo visual". \\
\hline Creatividad & $\begin{array}{l}\text { "La creatividad es un factor importante } \\
\text { al momento de idear una clase". }\end{array}$ \\
\hline
\end{tabular}

Se entiende que el uso de esta herramienta facilita la interacción con los estudiantes, pues el proceso de aprendizaje es muy dinámico, además fomenta su creatividad y les permite ser más autónomos. Los estudiantes tienen una mayor comprensión de los conocimientos impartidos por el docente cuando se 
implementa esta herramienta dentro de las clases. Ver TABLA II

TABLA II

TIPOS DE JUEGOS MÁS UTILIZADOS EN CLASE

\begin{tabular}{|c|c|}
\hline Términos & \multicolumn{1}{c|}{ Citas } \\
\hline Arte & $\begin{array}{c}\text { "Los ejercicios relacionados a cultura } \\
\text { Mrtística". }\end{array}$ \\
\hline Música & $\begin{array}{c}\text { "Los de encontrar las parejas de palabras o } \\
\text { figuras y las dinámicas musicales". } \\
\text { "Los ejercicios que tienen gráficos o los que } \\
\text { usan sonidos en las imágenes". }\end{array}$ \\
\hline Lenguaje & $\begin{array}{c}\text { "Los de encontrar las parejas de palabras o } \\
\text { figuras y las dinámicas musicales". } \\
\text { "Les gusta leer el contenido de las fichas } \\
\text { porque justamente recién están aprendiendo a } \\
\text { leer, a escribir manuscrito y con el teclado, } \\
\text { entonces son muy activos con la lectura". }\end{array}$ \\
\hline Visuales & $\begin{array}{c}\text { "Los ejercicios que tienen gráficos o los que } \\
\text { usan sonidos en las imágenes". } \\
\text { "Juegos en los que tengan que interactuar, } \\
\text { Interacción } \\
\text { puedandenasosiar fácilmente". }\end{array}$ \\
\hline
\end{tabular}

Los juegos son una buena herramienta para que los niños aprendan de una forma divertida, los tipos de juegos por el que los estudiantes se sienten más atraídos son aquellos que tienen una interfaz interactiva y que están relacionados con el arte, música y que sean visualmente estéticos. Ver TABLA III

TABLA III

DESVENTAJAS SEGÚN PROFESORES

\begin{tabular}{|c|l|}
\hline Dificultad & $\begin{array}{l}\text { "Mayor dificultad es la falta de equipos } \\
\text { tecnológicos, conexión y falta de apoyo de } \\
\text { los padres. No repaso entre alumnos". }\end{array}$ \\
\hline Desconocimiento & $\begin{array}{l}\text { "La falta de recursos y de conocimiento } \\
\text { entorpece grandemente la educación por el } \\
\text { medio digital, como docente estamos } \\
\text { acostumbrados más a lo tradicional y el } \\
\text { desconocimiento del uso de herramientas } \\
\text { ha sido el factor determinante para } \\
\text { entorpecer nuestro trabajo". }\end{array}$ \\
\hline Costumbre & $\begin{array}{l}\text { "La falta de recursos y de conocimiento } \\
\text { entorpece grandemente la educación por el } \\
\text { medio digital, como docente estamos } \\
\text { acostumbrados más a lo tradicional y el } \\
\text { desconocimiento del uso de herramientas } \\
\text { ha sido el factor determinante para } \\
\text { entorpecer nuestro trabajo". }\end{array}$ \\
\hline Dependencia & $\begin{array}{l}\text { "Los estudiantes no son } \\
\text { independientes". }\end{array}$ \\
\hline Accesibilidad & $\begin{array}{l}\text { "Deberían ser las herramientas } \\
\text { accesibles para toda clase social, en estos } \\
\text { momentos solo grandes instituciones de } \\
\text { elite tienen el acceso que debe ser para } \\
\text { todos y no para un sector en específico". }\end{array}$ \\
\hline
\end{tabular}

Los comentarios que más se repiten en la aplicación de esta pregunta, indican que las dificultades a la hora de utilizar este tipo de herramientas son la falta de recursos y conocimientos sobre su uso, ya que en Ecuador no son métodos que se acostumbren a utilizar. Ver TABLA IV

TABLA IV

TIPOS DE JUEGOS MÁS UTILIZADOS EN CLASE

\begin{tabular}{|c|l|}
\hline Complemento & $\begin{array}{l}\text { "Considero que ambas se } \\
\text { complementan, lo digital y tradicional } \\
\text { juntos son el método ideal". }\end{array}$ \\
\hline Adaptación & $\begin{array}{l}\text { "Para trabajar con mis niños de 5-6 años } \\
\text { prefiero el tradicional porque resulta mejor } \\
\text { que la maestra esté presente con el } \\
\text { estudiante". } \\
\text { "Considero que como docente debemos } \\
\text { adaptarnos a todo, debemos buscar la } \\
\text { manera de que el niño aprenda, en este } \\
\text { mundo que cada día va evolucionando } \\
\text { debemos estar listos para lo que venga". }\end{array}$ \\
\hline Empatía & $\begin{array}{l}\text { "Preferiría la educación tradicional ya } \\
\text { que por este medio puedo tener una empatía } \\
\text { directa con el alumno, el saber cuáles son } \\
\text { los puntos clave para llegar a él y el } \\
\text { conocimiento adquirido perdure". }\end{array}$ \\
\hline Conocimiento & $\begin{array}{l}\text { "Preferiría la educación tradicional ya } \\
\text { que por este medio puedo tener una empatía } \\
\text { directa con el alumno, el saber cuáles son } \\
\text { los puntos clave para llegar a él y el } \\
\text { conocimiento adquirido perdure". }\end{array}$ \\
\hline
\end{tabular}

Aunque el método tradicional ayuda a que los docentes desarrollen empatía con los estudiantes más fácilmente, los métodos digitales complementan el desarrollo interactivo y dinámico de una clase.

\section{Encuestas}

\section{1) Niños}

Nuestra consigna de encuesta se basó en conocer los criterios de los niños $(n=55)$ acerca de los métodos tradicionales y digitales de dar la clase, así como su actitud sobre las pizarras digitales en conjunto a los juegos. En la Fig. 1 Podemos notar que el $87 \%$ conoce juegos educativos, mientras que el $13 \%$ no.

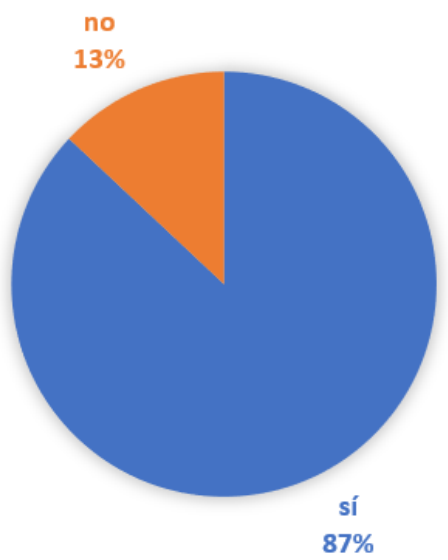

Fig. 1 Gráfico de pastel sobre conocimiento de juegos educativos

Siendo la Fig. 2, que muestra la materia que desearían aprender más con esta modalidad, el área de Matemáticas (38\%), Inglés (27\%), y Ciencias con un $18 \%$. 
Materias que les gustaría aprender mediante juegos virtuales

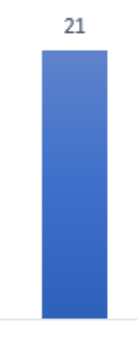

Matemáticas
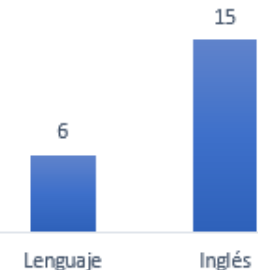

Inglés

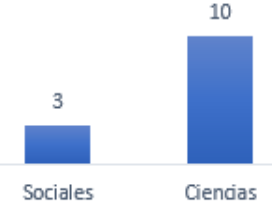

Sociales
Fig. 2 Gráfico de columnas sobre preferencia de materias en juegos educativos

En la Fig. 3, se puede ver que, aunque el $65 \%$ de niños no han recibido clases con pizarras digitales, consideran que son muy útiles y fáciles de usar, pues los ayudaría a motivarse durante sus clases.

\section{CLASES CON PIZARRAS DIGITALES}

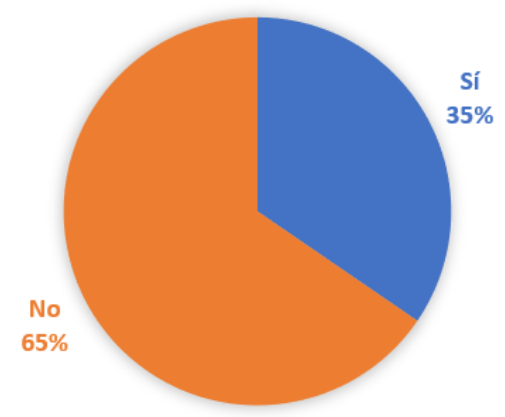

Fig. 3 Gráfico de pastel sobre clases con pizarras digitales

Así mismo, prefieren en general, clases que tengan este tipo de nueva modalidad para romper el molde de las clases tradicionales del día a día. Siendo un $84 \%$ quienes prefieren las clases digitales con nuevas tecnologías como las pizarras virtuales, independiente si son en línea o presenciales, que a una clase con pizarra normal (Fig. 4).

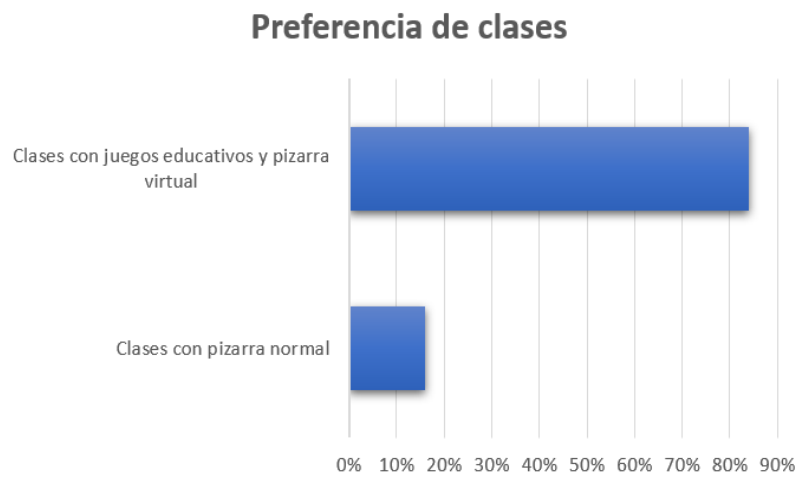

Fig. 4 Gráfico de barras sobre preferencia de modalidad de clases

\section{2) Profesores}

En las encuestas a profesores, se puede notar que el $96 \%$ sí conocen las pizarras digitales y piensan que optimizan la clase y promueven la participación. Pero se encuentran en un conflicto, al dudar del manejo adecuado de la herramienta. Ver Fig. 5

\section{CONOCIMIENTO DE EXISTENCIA DE PIZARRAS VIRTUALES}

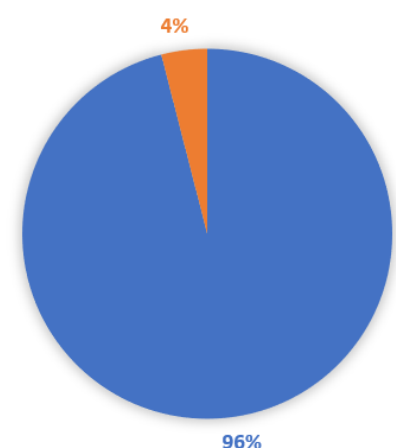

Fig. 5 Gráfico de pastel sobre conocimiento de pizarras virtuales

En la Fig. 6, se muestra que existe división en los porcentajes del uso de las pizarras. La mitad de los profesores no se sienten muy seguros al usar estas herramientas digitales. Interpretando los datos con los obtenidos en la entrevista, uno de los factores influyentes pudiese ser la curva de aprendizaje, propia de la edad de los docentes.

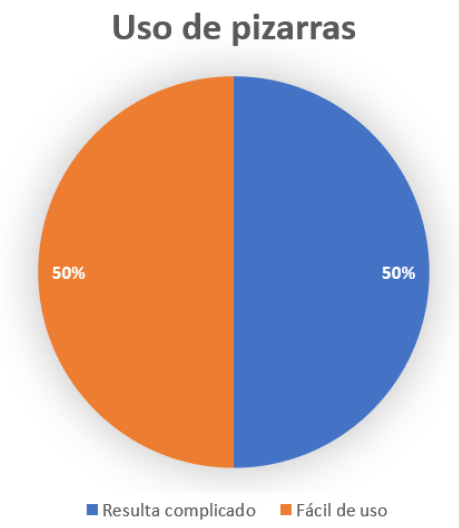

Fig. 6 Gráfico de pastel sobre uso de pizarras virtuales

El $42 \%$ de los profesores comentan que este tipo de herramienta llega a comunicar hasta un 4 sobre 5 puntos de excelencia de comunicación en el área de los objetivos de la clase, mientras el 38\% establece que llega a un 5 sobre 5 puntos de excelencia de comunicar los objetivos de la clase, dejando un aprendizaje de medio a alto. Así como los niños, los profesores prefieren el método digital para poder desarrollar sus clases, con el $17 \%$ prefiriendo el método tradicional. Ver Fig. 7

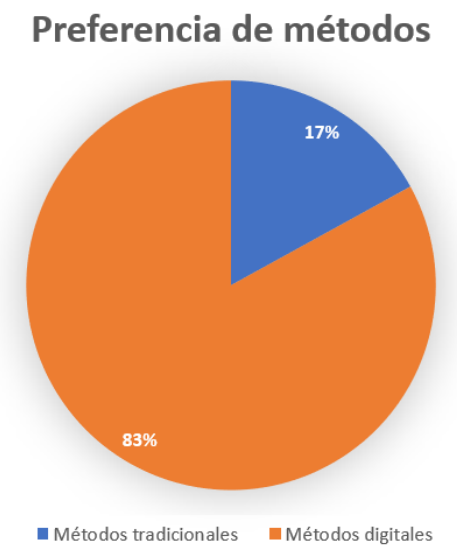

Fig. 7 Gráfico de pastel sobre preferencia de modalidad 
Finalmente, en la Figura 8, se puede ver que solo la mitad de quienes contestaron las encuestas conoce juegos en conjuntos de pizarra y por medio de una pregunta abierta, resalta la consistencia de la herramienta Kahoot.

\section{CONOCIMIENTO EN EXISTENCIA DE JUEGOS CON PIZARRAS VIRTUALES}

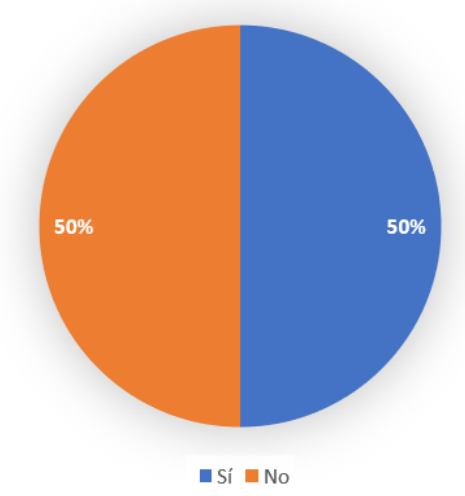

Fig. 8 Gráfico de pastel sobre uso de juegos educativos más pizarras virtuales

\section{CONCLUSIONES}

Esta investigación fue realizada con el propósito de conocer la opinión y criterio de estudiantes y profesores en las modalidades de planificar y dictar clases. Se destaca que la mayoría de los profesores, tanto en entrevistas como en las encuestas afirma que el método tradicional y el método digital deben ir de la mano, ya que estamos en un mundo cambiante, en donde, predomina el componente tecnológico.

Las clases siempre deben ir más allá, debido a que, en la sociedad actual, lo visual predomina al momento de la enseñanza. Además, aplicado en el contexto de la situación actual del Covid19, esta forma de ofrecer clases ayuda a romper el molde del día a día de una clase virtual. Así mismo, las clases deben desarrollarse de manera interactiva en donde el alumno pueda demostrar lo aprendido por medio de juegos, gráficos y demás.

La mayoría de los profesores conocían las pizarras virtuales, pero la mitad no incorporaba juegos en esta, y se sentían inseguros al utilizar la herramienta, ya que, sitúan las clases como impredecibles, debido a las eventualidades como el tiempo insuficiente para realizar todas las actividades planeadas y la pérdida de conectividad de Internet que realiza un corte en el flujo de la clase. En cambio, los niños conocen juegos educativos, pero solo el $35 \%$ ha recibido clases con pizarras digitales. Aun así, ellos prefieren esta herramienta, y se sentirían motivados si los profesores la utilizarán más en sus clases. También, piensan que es de fácil uso e interacción y desearían que fueran implementadas en materias como matemáticas, lenguaje, ciencias e inglés. La diferencia de confianza en el uso de las pizarras es parte de la brecha tecnológica que existe entre las generaciones de estudiantes-profesores, aunque los profesores afirman estar preparados constantemente para cada clase. Utilizar otra forma de enseñanza en una clase virtual requiere de mucho esfuerzo $\mathrm{y}$ ayuda de los mismos estudiantes para que funcione.
Por ende, las pizarras virtuales se convierten en una herramienta completa y de gran ayuda a la hora de impartir conocimientos, tanto durante las clases sincrónicas como asincrónicas, en modalidades que requieren elementos lúdicos o de control de conocimientos formales, aplicables a niños de primeros años de escolaridad. Se vuelven un aliado en el desarrollo de actividades en el entorno de aislamiento que están experimentando los niños.

A través de las encuestas realizadas se dedujo que estas herramientas digitales deben ser de fácil acceso y manejo, tanto para estudiantes como para docentes. Así, como también debe existir la debida capacitación sobre su incorporación en la construcción de objetos de aprendizaje. Logrando estos aspectos predominantes, se podrá obtener buenos resultados en la enseñanza de los niños de educación básica.

\section{RECONOCIMIENTOS}

Este trabajo forma parte de una investigación realizada en el marco de la materia "Investigación Aplicada al Diseño" de la Facultad de Arte Diseño y Comunicación Audiovisual de la Escuela Superior Politécnica del Litoral, ESPOL, dictada y en colaboración con la PhD. Nayeth Solórzano como editores. Los autores de este artículo también desean expresar su agradecimiento. A los estudiantes Melanie Almeida, Sara Morante y Joselyn Vásquez del grupo de trabajo que colaboraron en el levantamiento de información. A docentes que participaron en las entrevistas realizadas, así como los padres de familia con sus hijos que participaron en las encuestas sobre esta investigación.

\section{REFERENCIAS}

[1] M. d. S. Pública, "Boletines epidemiológicos coronavirus por semanas," Sep. 29, 2020. [Online]. Available: https://www.salud.gob.ec/boletines-epidemiologicos-coronaviruspor-semanas/

[2] M. d. Educación, "Plan Educativo "Aprendamos juntos en casa'," Sep. 29, 2020. [Online]. Available: https://educacion.gob.ec/planeducativo-aprendamos-juntos-en-casa

[3] M. d. Educación, "Visualizador Estadístico," Sep. 29, 2020. [Online]. Available: https://educarecuador.gob.ec/visualizadorestadistico/

[4] E. Dans, "Enrique Dans," 2020. [Online]. Available: https://www.enriquedans.com/2020/04/la-ensenanza-online-yano-es-una-opcion.html .[Accesed: Nov. 03, 2020].

[5] E. Dans, "RUSC University \& Knowledge Sociel Journal," Feb. 2009. [En línea]. Available: http://dx.doi.org/10.7238/rusc.v6i1.26 .[ Accesed: Jun. 23, 2020].

[6] F. G. Barrio and V. Ballesteros Ávila, "El uso de herramientas 2.0 como recursos innovadores en el aprendizaje de niños y niñas en educación infantiI. Un estudio de caso de investigación-acción," EDUTEC: Revista Electrónica de Tecnología Educativa, $\mathrm{n}^{\circ} 48, \mathrm{p}$. 11, Junio 2014.

[7] X. A. Rodríguez, "Ventajas y desventajas de las clases virtuales," El Telégrafo, Jun. 17, 2020. [Online]. Available: https://www.eltelegrafo.com.ec/noticias/sociedad/6/clasesvirtuales

[8] C. X. Banchón Nieto, "Beneficios de estimulación cognitiva en niños con Síndrome de Down estudio a realizarse en Sociedad Ecuatoriana de Rehabilitación de los Lisiados (SERLI)," Guayaquil, 2020.

[9] D. Melo-Solarte y P. Díaz, "El Aprendizaje Afectivo y la Gamificación en Escenarios de Educación Virtual," Información

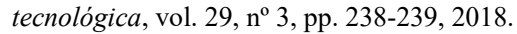

[10] M. Salazar, "De la tiza y la pizarra a las interacciones en el aula virtual," 2017. 
[11] R. M. Carro, A. M. Breda, G. Castillo and A. L. Bajuelos, "Generación de Juegos Educativos Adaptativos," de III Congreso Internacional de Interacción Persona-Ordenador, España, 2002.

[12] Redacción, "La reinvención de las reuniones en línea durante la pandemia," El Universo, Jun. 25, 2020. [Online]. Available: https://www.eluniverso.com/larevista/2020/06/25/nota/7884183/r einvencion-reuniones-linea-durante-pandemia .[Accesed: 2020].

[13] Redacción, «Alternativas para que docentes apliquen en la enseñanza virtual,» El Universo, Jun. 18, 2020. [Online]. Available:

https://www.eluniverso.com/noticias/2020/06/18/nota/7876098/al ternativas-que-docentes-apliquen-ensenanza-virtual .[Accesed: 2020].

[14] F. G. Barrio and V. Ballesteros, "El uso de herramientas 2.0 como recursos innovadores en el aprendizaje de niños y niñas en educación infantiI. Un estudio de caso de investigación-acción," EDUTEC. Revista Electrónica de Tecnología Educativa., nº 48, pp. 8-9, 2014.

[15] M. Torres, "Coronavirus: Docentes adecúan las salas de sus casas en aulas virtuales," Expreso, May. 19, 2020. [Online]. Available: https://www.expreso.ec/guayaquil/coronavirus-docentes-adecuansalas-casas-aulas-virtuales-11627.html .[Accesed: Jun. 12, 2020].

[16] ÁrbolABC, "Portal Educativo para niños," Rainbow Tree Inc, 2020. [Online]. Available: https://arbolabc.com/ .[Accesed: Jun. $12,2020]$.

[17] P. Torres and J. K. Cobo, "Tecnología educativa y su papel en el logro de los fines de la educación," Educere: La Revista Venezuela de la Educación, vol. 21, nº 68, pp. 31-40, Jan. 182017

[18] A. Findeli, "Research Through Design and Transdisiplinarity: A Tentative Contribution to the Methodology of Design Reseach," Academia.edu, pp. 70-72, May. 2008.

[19] A. H. Galvis, "Oportunidades Educativas de las TIC," DocPlayer, pp. 4-5, Jun. 2004.

[20] J. T. Fouts, "Research on Computers and Education: Past, Present and Future," CiteSeerX, p. 60, Feb. 2000.

[21] C. Chaparro, G. Escalante and E. Samacá, "Las TIC como estrategia didáctica dentro del proceso educativo de estudiantes universitarios sordos," Cuidado y Ocupación Humana, vol. 2, p. 9 , 2019.

[22] ONU, "Recomiendan que los niños no usen pantallas hasta los dos años," UN News, Apr. 24, 2019. [Online]. Available: https://news.un.org/es/story/2019/04/1454801\#: :text=Los\%20ni \%C3\%B1os\%20menores\%20de\%20dos,mucho\%20una\%20hora $\% 20 \mathrm{al} \% 20 \mathrm{~d} \% \mathrm{C} 3 \% \mathrm{ADa}$.[Accesed: Aug. 2020].

[23] M. López, "Los colegios no deberían existir," Kindsein Magazine $n^{\circ} 21$, Jun. 2007.

[24] K. Robinson and L. Aronica, El Elemento, Segunda ed., Barcelona: Grijalbo, 2009

[25] R. Cala, L. I. Díaz, N. Espí and J. M. Tituaña, "El Impacto del Uso de Pizarras Digitales Interactivas (PDI) en el Proceso de Enseñanza Aprendizaje. Un Caso de Estudio en la Universidad de Otavalo," Scielo, vol. 29, $\mathrm{n}^{\circ}$ 5, 2018.

[26] R. Rosas, C. P. Pérez-Salas and P. Olguín, "Pizarras Interactivas para un aprendizaje motivado en niños con parálisis cerebral," Scielo, vol. 36, n 2, pp. 191-209, Sep. 2010.

[27] J. S. Beach, "Interactive Whiteboard Transition: A Case Study," TRACE Tennesse Reseach and Creative Exchange, May. 2012.

[28] J. Moscoso, "Los métodos mixtos en la investigación en educación: hacia un uso reflexivo," Cadernos de Pesquisa, vol. 47, $\mathrm{n}^{\mathrm{o}} 164$, pp. 5-6, 2017.

[29] N. D. Suárez-Montes, J. V. Sáenz-Gavilanes and J. M. MeroVélez, "Elementos esenciales del diseño de la investigación. Sus características.," Dominio de las Ciencias, vol. 2, nº 3, pp. 83-84, 2016.

[30] G. Ledesma, L. Rodríguez, M. Lazo and M. D. L. M. Calderón, "Sistema de tareas docentes interdisciplinarias para contribuir al aprendizaje de los métodos estadísticos," Gaceta Médica Espirituana, vol. 18, $\mathrm{n}^{\circ}$ 2, p. 5, 2016. 